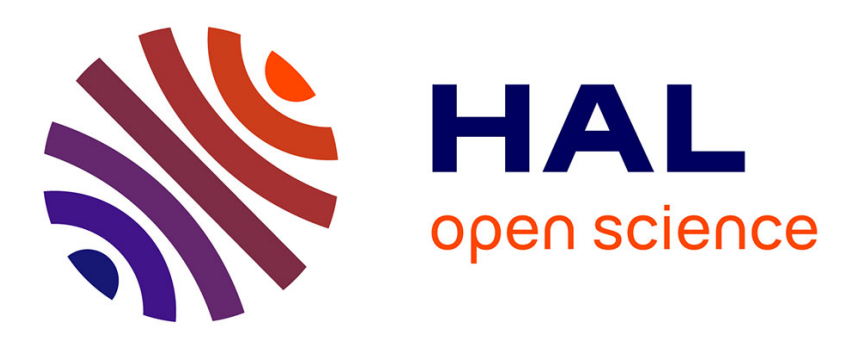

\title{
WEBEXPIR: Windowless Target Electron Beam Experimental Irradiation
}

\author{
Jan Heyse, Hamid Aït Abderrahim, Thierry Aoust, Marc Dierckx, Kris \\ Rosseel, Paul Schuurmans, Katrien van Tichelen, Arnaud Guertin, \\ Jean-Michel Buhour, Arnaud Cadiou, et al.
}

\section{To cite this version:}

Jan Heyse, Hamid Aït Abderrahim, Thierry Aoust, Marc Dierckx, Kris Rosseel, et al.. WEBEXPIR: Windowless Target Electron Beam Experimental Irradiation. HPPA 2007 - Fifth International Workshop on Utilisation and Reliability of High Power Proton Accelerators, May 2007, Mol, Belgium. pp.205-212. hal-01084114

\section{HAL Id: hal-01084114 https://hal.science/hal-01084114}

Submitted on 29 Jan 2021

HAL is a multi-disciplinary open access archive for the deposit and dissemination of scientific research documents, whether they are published or not. The documents may come from teaching and research institutions in France or abroad, or from public or private research centers.
L'archive ouverte pluridisciplinaire HAL, est destinée au dépôt et à la diffusion de documents scientifiques de niveau recherche, publiés ou non, émanant des établissements d'enseignement et de recherche français ou étrangers, des laboratoires publics ou privés. 


\title{
WEBEXPIR: WINDOWLESS TARGET ELECTRON BEAM EXPERIMENTAL IRRADIATION
}

\author{
Jan Heyse, Hamid Aït Abderrahim, Thierry Aoust, Marc Dierckx, Kris Rosseel, Paul \\ Schuurmans, Katrien Van Tichelen \\ SCK・CEN, Boeretang 200, BE-2400 Mol, Belgium \\ Arnaud Guertin, Jean-Michel Buhour, Arnaud Cadiou \\ SUBATECH, Ecole des Mines, 4 rue Alfred Kastler, BP 20722, F-44307 NANTES CEDEX 3 \\ Michel Abs, Benoit Nactergal, Dirk Vandeplassche \\ IBA, Chemin du Cyclotron 3, BE-1348 Louvain-la-Neuve, Belgium
}

\begin{abstract}
The WEBEXPIR (Windowless target Electron Beam EXPerimental IRradiation) program was set-up as part of the MYRRHA/XT-ADS R\&D efforts on the spallation target design, in order to answer different questions concerning the interaction of a proton beam with a liquid Lead-Bismuth Eutectic (LBE) free surface. An experiment was conceived at the IBA TT-1000 Rhodotron, a $7 \mathrm{MeV}$ electron accelerator which produces beam currents of up to $100 \mathrm{~mA}$. Due to the small penetration depth of the $7 \mathrm{MeV}$ electron beam and the high beam currents available, the TT-1000 allows to imitate the high power deposition at the MYRRHA/XT-ADS LBE free surface. The main goals of the experiment were to assess possible free surface distortion or shockwave effects in nominal conditions and during sudden beam on/off transient situations, as well as possible enhanced evaporation. The geometry and the LBE flow characteristics in the WEBEXPIR setup were made as representative as possible of the actual situation in the MYRRHA/XT-ADS spallation target. Irradiation experiments were carried out at beam currents of up to $10 \mathrm{~mA}$, corresponding to 40 times the nominal beam current necessary to reproduce the MYRRHA/XT-ADS conditions. As a preliminary general conclusion, it can be stated that the WEBEXPIR free surface flow was not disturbed by the interaction with the electron beam and that vacuum conditions stayed well within the design specifications.
\end{abstract}




\section{Introduction}

The design of the MYRRHA/XT-ADS, the European eXperimental Accelerator Driven System for the demonstration of Transmutation [1], includes a high power windowless spallation target operating with liquid Lead-Bismuth eutectic (LBE) that will be irradiated with a $600 \mathrm{MeV}$ proton beam at currents of up to $4 \mathrm{~mA}$. When considering such a high power windowless target design, a number of questions need to be addressed, such as the stability of the free surface flow and its ability to remove the power deposited by the proton beam by (forced) convection, the compatibility of a large hot LBE reservoir with the beam line vacuum and the outgassing of the LBE in the spallation target circuit. These issues have been studied during previous experiments supported by numerical simulations [1].

Another crucial point in the development of the spallation target is the demonstration of safe and stable operation of the free LBE surface under the irradiation with a high power proton beam. As a first step in this program, the WEBEXPIR (Windowless target Electron Beam EXPerimental IRradiation) experiment was set-up. Its purpose was to investigate the influence of LBE surface heating caused by a charged particle beam in a situation representative of the MYRRHA/XT-ADS. For this purpose a compact LBE loop was constructed with flow characteristics and geometry at the irradiation point that are very similar to the MYRRHA/XT-ADS case. The loop was connected to a 7 $\mathrm{MeV}$ electron accelerator. During irradiation the effects of the surface power deposition on the flow stability and LBE evaporation were monitored.

\section{Experimental setup}

\section{Interaction chamber}

The WEBEXPIR interaction chamber was constructed in such a way as to have interaction conditions that are very representative of the conditions in the actual MYRRHA/XT-ADS target nozzle (see Fig. 1). In MYRRHA/XT-ADS the proton beam passes through the inner of two hollow concentric vertical cylinders with the LBE flowing in between the inner and the outer cylinder. At the bottom of this downcomer section, the outer cylinder changes into a nozzle, resulting in a confluent LBE target. In order to avoid hitting the LBE recirculation zone which is present in the centre of the nozzle, the proton beam is painted along a circular path in between the recirculation zone and the inner cylinder, forming an annulus shaped footprint.

To simulate these conditions during the WEBEXPIR experiment, a geometry that can be seen as an unfolded slice of the MYRRHA/XT-ADS nozzle was chosen. It consists of a rectangular slide with its thickness corresponding to the nozzle gap thickness of $14.35 \mathrm{~mm}$ and with an angle of inclination of $26^{\circ}$, equal to angle between the proton beam and the LBE surface. The width of the gap was taken such that the LBE flow velocity was almost constant at the beam interaction point and equal to the MYRRHA/XT-ADS nozzle velocity of $2.5 \mathrm{~m} / \mathrm{s}$.

The part of the slide upstream of the interaction point is rib-roughened to prevent fluid acceleration and tearing. At the beam interaction point, the LBE flow is confined so that the impact of the beam can only be compensated in the direction perpendicular to the free surface. 
Figure 1: Schematic cutaway view of the MYRRHA/XT-ADS spallation target and core

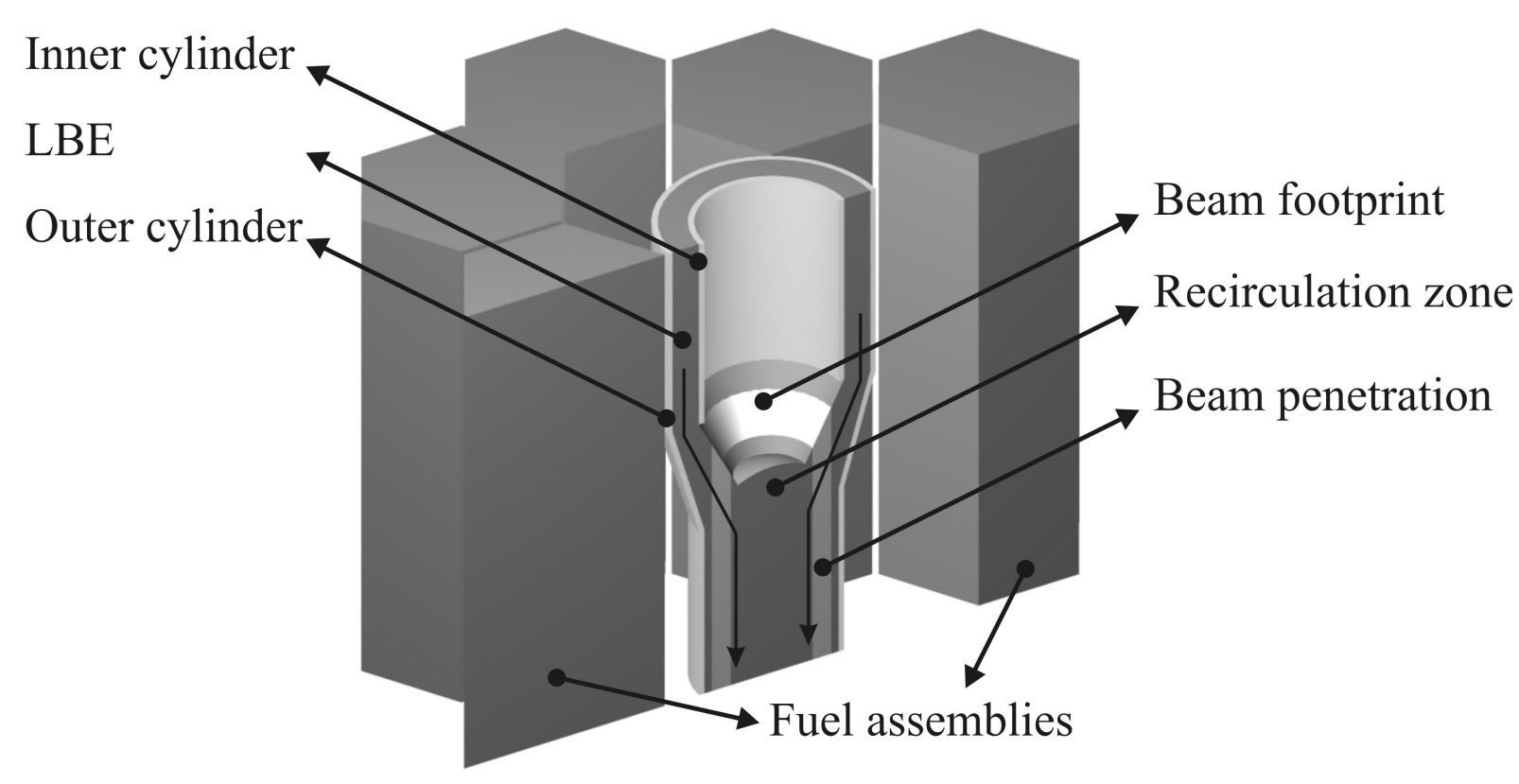

\section{Loop layout}

The interaction chamber was integrated in a compact LBE loop in order to achieve flow characteristics at the beam interaction point that were comparable to those in the MYRRHA/XT-ADS spallation target design. Apart from that, a number of spatial boundary conditions imposed by the configuration at IBA had to be taken into account.

Figure 2 shows a schematic drawing of the loop. The LBE is circulated by a 10 1/s, 5 bar magnetically coupled centrifugal pump (1). Because of space considerations, the LBE/oil heatexchanger (2), which ensures evacuation of the heat deposited by the electron beam, has been positioned on the exit pressure side of the pump. A valve (3) has been placed behind the heatexchanger in order to assure a stable pressure at the exit of the pump. A vortex type flow meter (4), which requires sufficient inlet and outlet tube length to obtain the necessary accuracy, is connected to a box at the top of the interaction chamber slide (5). In the interaction chamber (6), a number of viewports have been provided to monitor the interaction of the electron beam with the LBE flow. At the drain of the interaction chamber an expansion volume (7) has been added to prevent the flooding of the interaction chamber when the pump is not running. The top of the interaction chamber is connected to the beam line (8), just at the exit of the $270^{\circ}$ magnet that bends the electron beam coming from the accelerator into the vertical direction. The connection consists of a flexible bellows, a gate valve and a beam collimator. Expansion joints have been incorporated at several positions in the loop to relieve thermal strain and to correct for misalignment errors. 
Figure 2: WEBEXPIR loop layout - numbers are referred to in the text

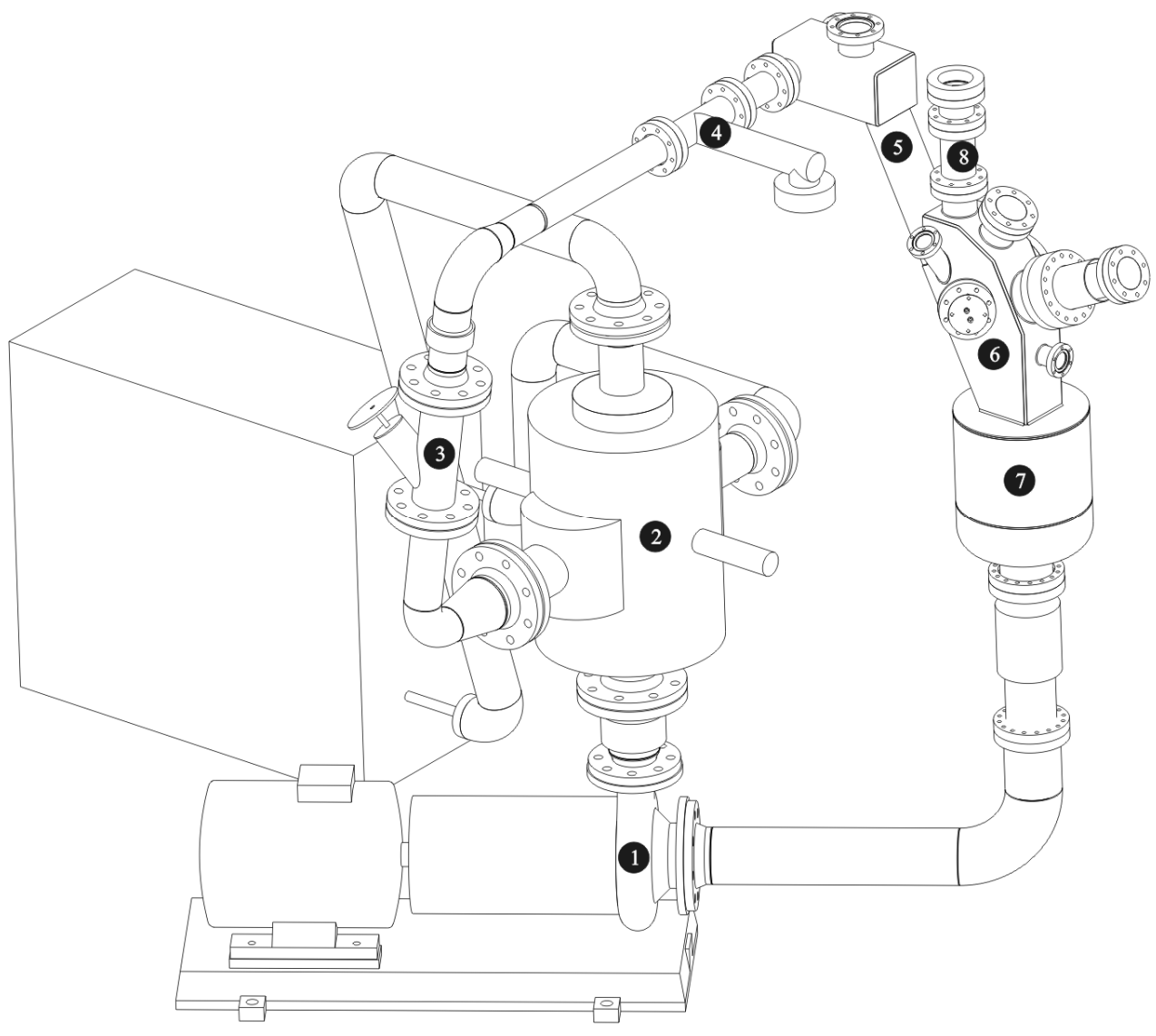

\section{Accelerator}

The irradiation was conducted at the TT-1000 Rhodotron [2], which is a very high power electron accelerator available at Ion Beam Applications s.a. (IBA, Louvain-la-Neuve, Belgium). Table 1 shows the main accelerator characteristics. The use of a $7 \mathrm{MeV}$ electron beam allows to imitate the effect of a 350 or $600 \mathrm{MeV}$ proton beam hitting the LBE surface. Moreover, the use of $7 \mathrm{MeV}$ electrons avoids problems with induced radioactivity, safety and waste that would occur when using protons.

Table 1: TT-1000 Rhodotron characteristics

\begin{tabular}{|l|l|}
\hline Energy & $7 \mathrm{MeV} \mathrm{e}$ \\
\hline Beam power range & $0.5-700 \mathrm{~kW}$ \\
\hline Beam spot & $10 \mathrm{~mm} \times 3 \mathrm{~mm}$ FWHM \\
\hline Beam entrance & Vertical from top \\
\hline Diameter & $3.0 \mathrm{~m}$ \\
\hline Height & $3.3 \mathrm{~m}$ \\
\hline
\end{tabular}


Figure 3 compares the linear power deposition in LBE for a proton beam with an energy of 350 $\mathrm{MeV}$ and $600 \mathrm{MeV}$, respectively [3], and an $7.5 \mathrm{MeV}$ electron beam, as calculated by Monte Carlo simulations. The inset of this figure clearly shows that the heating by the electron beam is concentrated near the lead bismuth surface: about $65 \%$ of the beam power is deposited in the first 2 $\mathrm{mm}$ below the LBE surface. This figure also shows that the linear power density per $\mathrm{mA}$ at the surface is well comparable to that of a $350 \mathrm{MeV}$ or $600 \mathrm{MeV}$ proton beam.

\section{Figure 3: Linear power deposition}

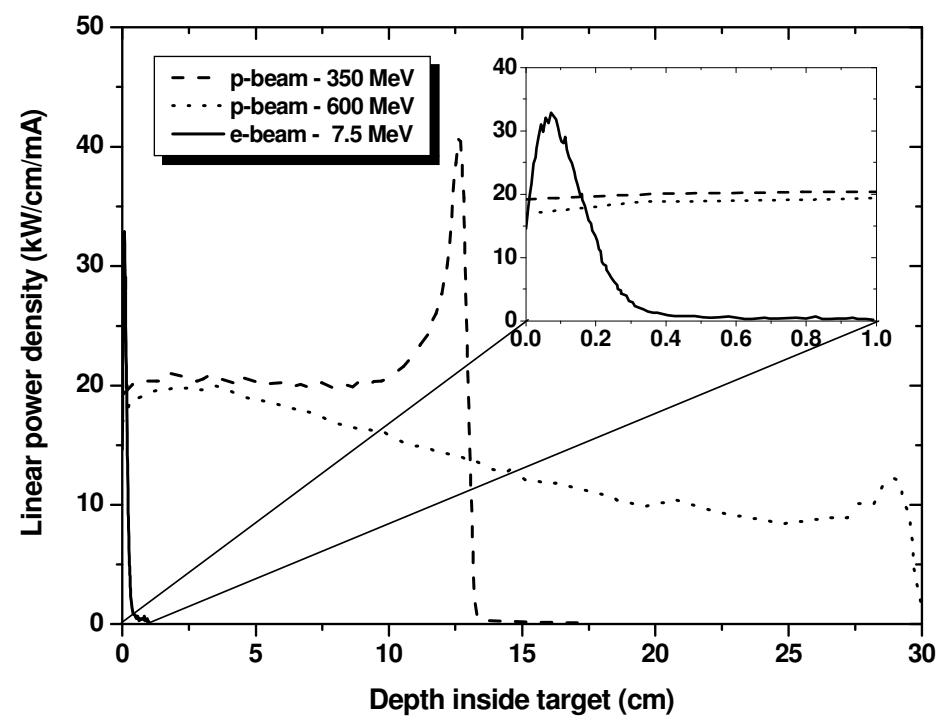

Table 2 compares the characteristics of the flow and the beam profile for the MYRRHA/XTADS and the WEBEXPIR case. In order to obtain at least the same surface heating conditions during the WEBEXPIR experiment, the nominal beam current was set to $0.25 \mathrm{~mA}$.

Table 2: Comparison of flow and beam characteristics

\begin{tabular}{|l|l|l|}
\hline & XT-ADS target & WEBEPXIR target \\
\hline Target material & LBE & LBE \\
\hline Beam energy & $600 \mathrm{MeV} \mathrm{p}$ & $7 \mathrm{MeV} \mathrm{e}-$ \\
\hline Average power deposition $<2 \mathrm{~mm}$ & $1.8 \mathrm{~kW} / \mathrm{mm} / \mathrm{mA}$ & $2.2 \mathrm{~kW} / \mathrm{mm} / \mathrm{mA}$ \\
\hline Flow velocity & $2.5 \mathrm{~m} / \mathrm{s}$ & $2.5 \mathrm{~m} / \mathrm{s}$ \\
\hline Beam shape & $\begin{array}{l}\sim \text { Gaussian }(10 \mathrm{~mm} \text { FWHM }) \\
\text { painted along annulus }(54 \mathrm{~mm} \varnothing)\end{array}$ & $\sim$ Gaussian $(3 \mathrm{~mm}$ FWHM $\perp$ flow, \\
& $4 \mathrm{~mA}$ & $\mathbf{0 . 2 5} \mathbf{~ m A}$ \\
\hline Beam current &
\end{tabular}

\section{Instrumentation}

A number of diagnostic devices have been installed on the interaction chamber in order to study the two main effects mentioned before, i.e. free surface distortion and enhanced evaporation. Additional devices were used to monitor the LBE and beam characteristics. 
- Optical camera: used for visual observation of the beam interaction point, both perpendicular and parallel to the LBE surface.

- Infrared camera: used for infrared observation of the beam interaction point and for measuring the surface heating at and downstream of the beam interaction point without interfering with the LBE flow.

- Thermocouple array: a set of thermocouples, inserted in the LBE flow downstream of the beam interaction point for measuring the surface heating caused by the beam.

- Pt-100 resistive sensors: used to accurately measure the LBE bulk temperature before and after beam interaction.

- Thermocouples on the loop: used to measure the temperature of the main loop components, the temperature of the top and sides of the interaction chamber and the temperature of the beam collimator.

- Dedicated nude active ionisation pressure gauge: used for determining the enhanced evaporation at the beam interaction point.

- Additional Bayard-Alpert pressure gauges: used for monitoring the vacuum conditions in the interaction chamber and the beam line.

- Pressure transducers: used to monitor shockwaves underneath the LBE flow and to measure the LBE pressure at the entrance and exit of the main pump.

- Flow meter: used to measure the LBE flow rate.

- Beam position monitor: $\mathrm{Al}_{2} \mathrm{O}_{3}$ coated and water cooled copper support, used to verify the position of the electron beam in between irradiations.

- Beam collimator current read-out: used to monitor which fraction of the beam is stopped by the beam collimator.

\section{Measurements and discussion}

A set of measurements were performed at different electron beam currents to determine the experimental conditions and to address the beam-LBE interaction issues mentioned before. A detailed analysis of these measurements is ongoing, the results of which will be reported in a forthcoming publication. Table 3 shows the nominal LBE conditions with the electron beam turned off.

Table 3: Nominal LBE conditions with beam off

\begin{tabular}{|l|l|}
\hline LBE flow & $3.8 \mathrm{l} / \mathrm{s}$ \\
\hline LBE speed at interaction point & $2.5 \mathrm{~m} / \mathrm{s}$ \\
\hline LBE bulk temperature & $200^{\circ} \mathrm{C}$ \\
\hline Total LBE mass in loop & $800 \mathrm{~kg}$ \\
\hline Vacuum in interaction chamber & $5 \times 10^{-6} \mathrm{mbar}$ \\
\hline
\end{tabular}

\section{Beam profile measurements}

The distribution of the current along the beam profile was determined prior to the actual irradiation experiments. For this, a stack of electrically insulated collimators with decreasing 
diameters was mounted at the exit of the $270^{\circ}$ bending magnet. The fraction of the beam current caught by each of the collimators was read out. The remaining fraction of the beam was deposited in a water cooled beam dump. Since the profile of the beam might change as a function of the total beam current, the beam profile was measured for currents of $100 \mu \mathrm{A}$ up to $4 \mathrm{~mA}$.

Apart from this, the beam position and profile were also visually checked by inserting an $\mathrm{Al}_{2} \mathrm{O}_{3}$ coated and water cooled copper support in the electron beam at the position of the beam interaction point (see Fig. 3). The interaction with the $7 \mathrm{MeV}$ electrons produces visual radiation which can be detected with an optical camera.

The combination of both measurements resulted in a beam profile with an average full width at half maximum (FWHM) of $10 \mathrm{~mm}$ in the direction parallel to the LBE flow and $3 \mathrm{~mm}$ perpendicular to the LBE flow.

Figure 4: Beam spot on $\mathrm{Al}_{2} \mathrm{O}_{3}$

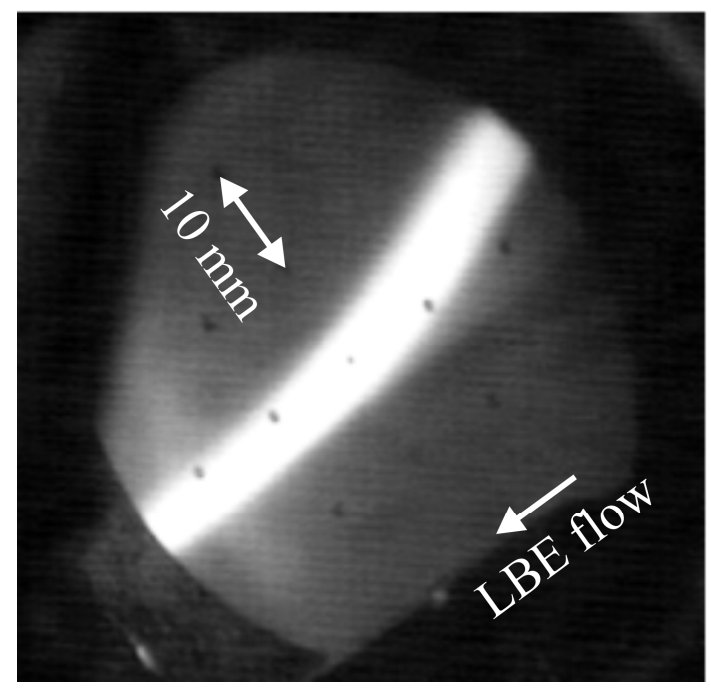

\section{Visual inspection}

An optical camera was positioned both nearly perpendicular and parallel to the LBE surface, focussing on the beam interaction point. Visual images were recorded for beam currents of $100 \mu \mathrm{A}$ up to $5 \mathrm{~mA}$. No obvious distortion of the LBE surface was detected for any of the beam currents.

\section{Infrared inspection}

The infrared camera was positioned perpendicular to the LBE surface, focussing on the LBE surface just downstream of the beam interaction point. The camera was used to measure the surface heating of the LBE for beam currents of $100 \mu \mathrm{A}$ up to $7.5 \mathrm{~mA}$. 


\section{Enhanced evaporation}

The enhanced evaporation of the LBE as a result of surface heating by the electron beam was measured with a nude Bayard-Alpert vacuum gauge positioned close to the beam interaction point. The increase in pressure was monitored for beam currents of $100 \mu \mathrm{A}$ up to $10 \mathrm{~mA}$. Even at $10 \mathrm{~mA}$, the pressure in the interaction chamber stayed well below $10^{-4}$ mbar.

Surface samples were taken at a number of cold positions in the interaction chamber for off-line determination of the amount of LBE condensation.

Figure 5: Infrared image of beam spot on LBE at a beam current of $1.5 \mathrm{~mA}$

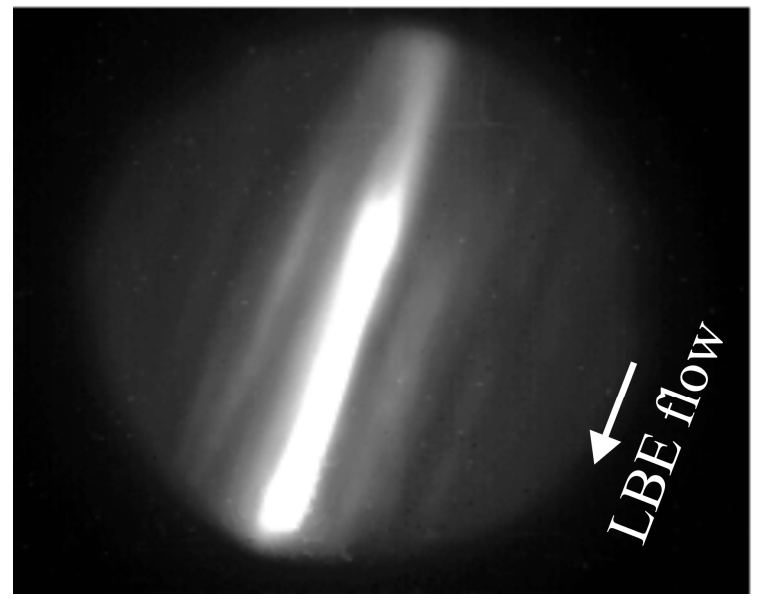

\section{Conclusion}

The aim of the WEBEXPIR experiment was to check for any LBE surface distortion or enhanced evaporation as a result of surface heating with a $7 \mathrm{MeV}$ electron beam in conditions representative of the MYRRHA/XT-ADS spallation target. Various tests have been carried out at electron beam currents of up to $10 \mathrm{~mA}$, which corresponds to 40 times the nominal beam current necessary to reproduce the MYRRHA/XT-ADS conditions.

No significant shockwave effects or enhanced LBE evaporation were detected. As a first preliminary conclusion it can be stated that the WEBEXPIR free surface flow was not disturbed by the interaction with the electron beam and that the vacuum conditions stayed well within the design specifications.

\section{REFERENCES}

[1] P. Schuurmans et al., Design and R\&D support of the XT-ADS Spallation Target Loop, these proceedings

[2] M. Abs, Y. Jongen, E. Poncelet and J. -L. Bol, Radiat. Phys. Chem. 71 1-2, pp 287-290 (2004)

[3] H. Aït Abderrahim et al., MYRRHA Draft 2, Chapter 4 Neutronics, RF\&M/EM/em.34.B043200/85/05-26 (2005) 